\title{
Estimulação diafragmática elétrica transcutânea (EDET) para fortalecimento muscular respiratório: estudo clínico controlado e randomizado
}

\author{
Transcutaneous electrical diaphragmatic stimulation (TEDS) for the respiratory \\ muscle strengthening: randomized and controlled clinical study \\ Karina Maria Cancelliero', Daniela Ike², Luciana Maria Malosá Sampaio³, Vera Lucia Alves dos Santos, \\ Roberto Stirbulov ${ }^{4}$, Dirceu Costa ${ }^{3,5}$
}

RESUMO I O objetivo do estudo foi demonstrar o efeito de dois protocolos da estimulação diafragmática elétrica transcutânea (EDET) sobre a força muscular respiratória de mulheres saudáveis, sendo um protocolo segundo Geddes et al. (1988) e outro padronizado pelo equipamento Phrenics. Mulheres saudáveis foram divididas em 3 grupos: Controle ( $n=7)$; EDET com Phrenics ( $n=7)$ e EDET com Dualpex ( $n=7$ ), sendo o tratamento realizado 2 vezes por semana, durante 6 semanas (12 sessões). Foram avaliadas a pressão inspiratória máxima (PImáx) e pressão expiratória máxima (PEmáx), antes e após o tratamento. A análise estatística foi realizada pelo teste Shapiro-Wilk e Kruskal Wallis com pós-hoc de Dunn $(p<0,05)$. Os dois grupos experimentais apresentaram aumento na PImáx (Phrenics: 32,9\%; Dualpex: 63,2\%) e na PEmáx (Phrenics: 44,7\%; Dualpex: 60,9\%), diferentemente do Controle que não apresentou diferença. Em conclusão, os dois protocolos de EDET promoveram aumento da força muscular inspiratória e expiratória em mulheres saudáveis.

Descritores | estimulação elétrica; músculos respiratórios; diafragma; força muscular; fisioterapia.
ABSTRACT I The objective of this study was to demonstrate the effect of two protocols of transcutaneous electrical diaphragmatic stimulation (TEDS) on the respiratory muscle strength of healthy women, with one protocol according to Geddes et al. (1988) and other protocol standardized by Phrenics equipment. Healthy women were divided in 3 groups: Control $(n=7)$; TEDS with Phrenics ( $n=7$ ) and TEDS with Dualpex $(n=7)$. The treatment was made twice a week, during 6 weeks (12 sessions). Respiratory muscle strength was evaluated by maximal inspiratory pressure (MIP) and maximal expiratory pressure (MEP) before and after the treatment. The statistical analysis was made by the Shapiro-Wilk and the Kruskal Wallis test with Dunn's post-hoc test $(p<0.05)$. Both TEDS protocols promoted increase in MIP (Phrenics: 32.9\%; Dualpex: 63.2\%) and in MEP (Phrenics: 44.7\%; Dualpex: 60.9\%), differently of the Control that didn't show difference. In conclusion, the two TEDS' protocols promoted increase of inspiratory and expiratory muscle strength in healthy women.

Keywords | electrical stimulation; respiratory muscles; diaphragm; muscle strength; physical therapy specialty.

\footnotetext{
Estudo desenvolvido no ambulatório de Fisioterapia da Faculdade Anhanguera (FAC) de Piracicaba - Piracicaba (SP), Brasil. 'Pós-doutoranda em Fisioterapia pelo Programa Nacional de Pós-Doutorado (PNPD), Programa de Pós-graduação em Fisioterapia (PPG-Ft) da Universidade Federal de São Carlos (UFSCar) - São Carlos (SP), Brasil.

2Doutoranda em Fisioterapia do PPG da UFSCar - São Carlos (SP), Brasil; Docente do curso de graduação em Fisioterapia da FAC de Piracicaba - Piracicaba (SP), Brasil.

${ }^{3}$ Docente do Programa de Pós-graduação em Ciências da Reabilitação (PPG-CR) da Universidade Nove de Julho (UNINOVE) - São Paulo (SP), Brasil.

${ }^{4}$ Docente do Departamento de Pneumologia da Santa Casa de Misericórdia de São Paulo - São Paulo (SP), Brasil.

${ }^{5}$ Docente do PPG em Ciências da Reabilitação da UNINOVE - São Paulo (SP), Brasil, Docente do PPG em Fisioterapia da UFSCar - São Carlos (SP), Brasil.
}

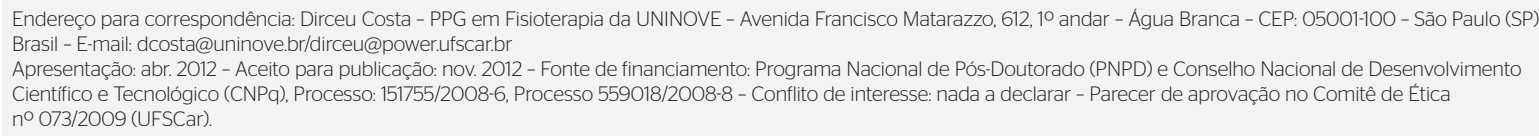




\section{INTRODUÇÃO}

Apesar de ainda haver escassez de estudos relacionados à estimulação diafragmática elétrica transcutânea (EDET), este recurso tem sido utilizado na prática clínica da fisioterapia. $\mathrm{Na}$ literatura sobre o tema, os efeitos da EDET estão relacionados com variáveis respiratórias ${ }^{1-5}$, sendo que os equipamentos ou os parâmetros da corrente elétrica se diferem na prática clínica, com a utilização de diferentes protocolos pelo profissional fisioterapeuta. Contudo, pouco tem sido explorado sobre os protocolos e metodologias de utilização da EDET.

Em alguns estudos, preconizou-se a utilização dos parâmetros da EDET segundo o protocolo do estudo de Geddes et al. ${ }^{2,6-8}$. Segundo esses estudos, os parâmetros para a aplicação da EDET em humanos são: corrente modulável com tempo de subida de $1 \mathrm{~s}$; tempo de sustentação da contração de $1 \mathrm{~s}$; tempo de relaxamento de 2 s; frequência da corrente entre 25 e $30 \mathrm{~Hz}$; largura de pulso entre 0,1 e $10 \mathrm{~ms}$; eletrodos fixados em pontos paraxifoideos ou intercostais na linha média axilar; intensidade mínima para obter a contração; tempo de estimulação de 20 min. Porém, conforme já exposto, na prática clínica da fisioterapia observam-se diferentes parâmetros, carecendo a comprovação científica da efetividade dos protocolos.

Foi nesse contexto que surgiu um equipamento de EDET, específico para a estimulação elétrica do diafragma, denominado Phrenics, com parâmetros padronizados e limitados, objetivando facilitar sua utilização nos pacientes com disfunção respiratória. Seus parâmetros, apesar de serem similares ao protocolo de Geddes et al., ${ }^{2,7}$, diferem em relação aos tempos de subida, sustentação e descida. Apesar de haver referência de estudos utilizando o Phrenics no tratamento com a $\mathrm{EDET}^{4,5}$, não foram encontrados na literatura estudos que compararam os diferentes protocolos, em especial esses dois mencionados.

Diante do exposto, o objetivo desse estudo foi demonstrar dois diferentes protocolos de EDET, um segundo Geddes et al. ${ }^{2,6-8}$ e outro proposto pelo fabricante do Phrenics especificamente sobre a força muscular respiratória de mulheres saudáveis, com a hipótese de que os dois protocolos melhoram a força muscular respiratória, independente dos tempos de subida, sustentação e descida, uma vez que o Phrenics foi desenvolvido especificamente para a contração do músculo diafragma.

\section{METODOLOGIA}

\section{Amostra}

Este estudo foi controlado e randomizado, sendo triadas 21 mulheres jovens saudáveis, recrutadas na faculdade de Fisioterapia. Foi realizada uma seleção, tomando-se como base os critérios de inclusão e exclusão, cujo número de indivíduos foi baseado na literatura ${ }^{9}$ e calculado para $7 \mathrm{em}$ cada grupo, com erro tipo I de 5\% e poder de $80 \%$.

Essas voluntárias foram divididas aleatoriamente em 3 grupos: EDET com Phrenics ( $n=7)$, EDET com Dualpex $(n=7)$ e Grupo Controle $(n=7)$. Foram excluídas mulheres com idade superior a 35 anos e inferior a 20 anos, com obesidade ou sobrepeso, e que apresentassem doenças pulmonares, cardiovasculares, neurológicas e ortopédicas, ou qualquer disfunção física que as impedisse de participar do estudo.

Este trabalho foi aprovado pelo Comitê de Ética em Pesquisa da Universidade (protocolo 073/2009) e as voluntárias assinaram o termo de consentimento livre e esclarecido, conforme determina a resolução 196/96 do Conselho Nacional de Saúde.

\section{Avaliação}

Inicialmente, foram verificadas a pressão arterial $(\mathrm{mmHg})$, saturação periférica de oxigênio (\%), frequência cardíaca (bpm), peso (kg), altura $(\mathrm{m})$ e índice de massa corporal (IMC).

Para a avaliação da força muscular respiratória, tanto a pressão inspiratória máxima (PImáx) quanto a pressão expiratória máxima (PEmáx), foi utilizado um manovacuômetro analógico $\left(\mathrm{Ger}-\mathrm{Ar}^{\circledR}\right)$ com adaptador de bocais, contendo um orifício de aproximadamente $2 \mathrm{~mm}$ de diâmetro, com a finalidade de proporcionar um escape de ar e assim prevenir a elevação da pressão gerada na cavidade oral, pelos músculos da parede bucal, evitando com isso a interferência nos resultados ${ }^{10,11}$. $\mathrm{O}$ intervalo operacional do aparelho foi de $0 \pm 300 \mathrm{cmH}_{2} \mathrm{O}$.

A PImáx foi mensurada a partir do volume residual, ou seja, ao término de uma inspiração máxima, partindo de uma expiração máxima, enquanto que a PEmáx foi mensurada a partir da capacidade pulmonar total, ou seja, ao término de uma expiração máxima, partindo de uma inspiração máxima ${ }^{12}$. Todas as voluntárias realizaram no mínimo três manobras reprodutíveis, tanto para a PImáx, quanto para a PEmáx, todas sustentadas por dois segun$\operatorname{dos}^{13}$, sendo que o maior valor foi computado, desde que 
este apresentasse $10 \%$ ou menos de diferença em relação ao valor imediatamente inferior. Todas as voluntárias permaneceram na posição sentada e fazendo uso de um clipe nasal.

Destaca-se que o avaliador não soube qual protocolo o voluntário recebeu quando realizou a reavaliação.

\section{Protocolo}

Para o procedimento da EDET foi utilizado o aparelho Dualpex, modelo Phrenics e o Dualpex 961, descrito e fundamentado segundo Geddes et al. ${ }^{2}$.

Ambos os equipamentos são da marca Quark $^{\circledR}$ (Brasil). O Phrenics, desenvolvido especificamente para a EDET, tem alguns parâmetros fixos e já modulados na corrente, e foi utilizado no grupo EDET com o Phrenics, sendo a corrente elétrica pulsada, bifásica e simétrica, com os seguintes parâmetros modulados: frequência de $30 \mathrm{~Hz}$; largura de pulso $(\mathrm{T})$ de $0,4 \mathrm{~ms}$, subida (rampa) de 0,7 s (valor máximo) e frequência respiratória de $15 \mathrm{rpm}$. A sustentação foi de $0,4 \mathrm{~s}$, já padronizada e fixa no aparelho. O Dualpex 961 permite ser modulado livremente e foi aplicado no grupo EDET com Dualpex, sendo que a corrente elétrica utilizada foi pulsada, bifásica e simétrica, com os seguintes parâmetros: frequência de $30 \mathrm{~Hz}$; T de 0,4 ms; rampa de $1 \mathrm{~s}$; sustentação de $1 \mathrm{~s}$ e descida de $2 \mathrm{~s}^{2}$. Sendo assim, a diferença entre os protocolos está nos tempos de subida e sustentação, além da descida não estar presente no Phrenics, ou seja, após a sustentação há o repouso.

Nos dois equipamentos, a intensidade da corrente foi a mínima para obter a con tração do músculo diafragma, causando sensação confortável à voluntária ${ }^{2,14}$. A colocação dos eletrodos foi realizada da seguinte maneira: 4 eletrodos de silicone-carbono com tamanhos $3 \times 5 \mathrm{~cm}$ (2 eletrodos) e 4,5x5 cm (2 eletrodos) adaptados na pele (previamente limpa com gel hidrossolúvel) com fita tipo micropore $\left(3 \mathrm{M}^{\circledR}\right)$. Foram posicionados dois eletrodos em cada lado do tórax, sendo um na região paraxifoidea direita e esquerda no terceiro espaço intercostal $(\mathrm{EIC})(3 \times 5 \mathrm{~cm})$ e o outro na linha axilar média, na região do sétimo EIC $(4,5 \times 5 \mathrm{~cm})$.

Cada sessão teve duração de $30 \mathrm{~min}$, com as voluntárias posicionadas em decúbito dorsal (semi-fowler), com os membros inferiores estendidos e braços ao longo do corpo. Foi orientado para que coordenassem a respiração com o disparo da corrente.

A análise da intervenção foi realizada antes da aplicação (1 semana antes do início) e após a intervenção (1 semana após o término das sessões). A duração foi de 6 semanas, sendo 2 vezes por semana, totalizando 12 sessões.

O grupo Controle não recebeu intervenção, sendo que a avaliação também foi realizada anterior e posterior as seis semanas. Tomou-se o cuidado para que as voluntárias deste grupo não tivessem conhecimento sobre o tipo de intervenção dos outros grupos.

\section{Tratamento dos dados}

Primeiramente foi aplicado o teste de normalidade Shapiro-Wilk e após o teste de Kruskal Wallis para a comparação intergrupos com pós-hoc de Dunn. Para essa análise utilizou-se também o delta de variação ((Pós-Pré/Pré)*100)). Para a análise dos dados antropométricos, foi utilizada a análise de variância (ANOVA) seguida do teste de Tukey para a comparação intergrupo. O nível de significância foi de $5 \%(\mathrm{p}<0,05)$.

\section{RESULTADOS}

De acordo com as características da amostra apresentadas na Tabela 1, pode-se constatar um bom nível de homogeneidade antropométrica, pois mesmo a saturação periférica de oxigênio $\left(\mathrm{SpO}_{2}\right)$, frequência cardíaca

Tabela 1. Características antropométricas dos grupos Controle, Phrenics e Dualpex

\begin{tabular}{|c|c|c|c|c|}
\hline Variável & Controle & Phrenics & Dualpex & Valor $\mathrm{p}$ \\
\hline Idade (anos) & $26,10 \pm 5,60$ & $23,00 \pm 3,80$ & $23,90 \pm 2,60$ & $>0,05$ \\
\hline Altura (m) & $1,61 \pm 0,05$ & $1,67 \pm 0,07$ & $1,60 \pm 0,04$ & $>0,05$ \\
\hline Peso (kg) & $63,70 \pm 12,80$ & $60,00 \pm 7,90$ & $57,70 \pm 13,90$ & $>0,05$ \\
\hline IMC $\left(\mathrm{kg} / \mathrm{m}^{2}\right)$ & $24,40 \pm 4,10$ & $21,50 \pm 1,80$ & $22,50 \pm 4,90$ & $>0,05$ \\
\hline $\mathrm{FC}(\mathrm{bpm})$ & $80,70 \pm 12,10$ & $72,90 \pm 9,40^{*}$ & $74,40 \pm 5,30$ & $*<0,05$ \\
\hline $\mathrm{SpO}_{2}(\%)$ & $97,60 \pm 1,00$ & $99,90 \pm 0,40^{\star \star *}$ & $99,00 \pm 0,80^{* \star}$ & ${ }^{* * *}<0,001 /{ }^{* *}<0,01$ \\
\hline PAS (mmHg) & $101,40 \pm 12,10$ & $115,70 \pm 7,90^{*}$ & $111,40 \pm 6,90$ & ${ }^{*}<0,05$ \\
\hline PAD (mmHg) & $62,90 \pm 4,90$ & $74,30 \pm 9,80^{*}$ & $68,60 \pm 9,00$ & $*<0,05$ \\
\hline
\end{tabular}

Média e desvio padrão; $n=7 /$ grupo; ${ }^{*}$ comparado ao grupo Controle; ${ }^{*} \mathrm{p}<0,05,{ }^{* *} \mathrm{p}<0,01,{ }^{* * *} \mathrm{p}<0,001$

EDET: estimulação diafragmática elétrica transcutânea; IMC: índice de massa corporal; FC: frequência cardíaca; SpO; saturação periférica de oxigênio; PAS: pressão arterial sistólica; PAD: pressão arterial diastólica 
(FC) e pressão arterial (PA) diferentes entre os grupos, todos os valores estão nos níveis de normalidade. Destaca-se o fato de não haver perda de pacientes ou de follow-up.

Os dois protocolos de EDET promoveram aumento na PImáx, sendo 32,9\% no Phrenics e 63,2\% no Dualpex, diferentemente do grupo Controle, o qual não apresentou aumento. De forma semelhante, constatou-se aumento da PEmáx com o tratamento pelo Phrenics em $44,7 \%$ e pelo Dualpex em $60,9 \%$, sem diferença no Controle (Tabela 2).

Ao se comparar o delta de variação entre os grupos, constatou-se que houve diferença entre o grupo Controle e os dois grupos experimentais, tanto para a PImáx como a PEmáx, sem diferença entre os grupos experimentais (Figura 1).

A intensidade da corrente variou entre as voluntárias e as sessões. Assim, a média \pm desvio padrão (DP) para o grupo Phrenics no $3^{\circ}$ EIC foi de $16,2 \pm 3,8$ e no 7 o EIC foi de 24,2 $\pm 5,0$. Para o grupo Dualpex foi de $15,4 \pm 3,0$ no $3^{\circ} \mathrm{EIC}$ e de $21,4 \pm 6,3$ no $7^{\circ}$ EIC. A intensidade foi particularizada para cada voluntária, respeitando-se a sensibilidade e não houve efeitos indesejados ou complicações.

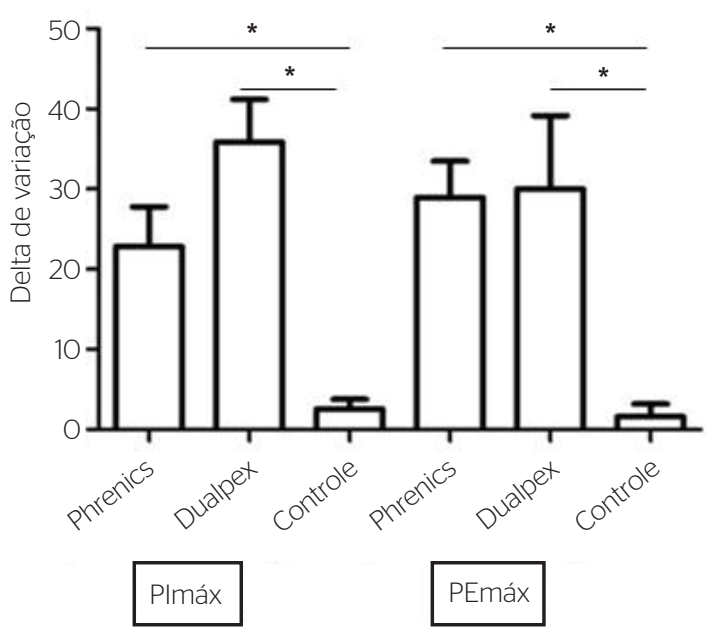

Média e desvio padrão; $n=7 / g r u p o ; ~ * p<0,05$ comparado à fase pré. PImáx: pressão inspiratória máxima; PEmáx: pressão expiratória máxima

Figura 1. Delta de variação das pressões respiratórias máximas dos grupos Controle, Phrenics e Dualpex

\section{DISCUSSÃO}

Os dois protocolos da EDET não se diferenciaram, mas tanto o Phrenics quanto o Dualpex melhoraram a força muscular respiratória, e assim, possivelmente, podem ser aplicados na prática da fisioterapia respiratória para pacientes que apresentem fraqueza muscular respiratória. Destaca-se que o aumento ocorreu tanto na PImáx quanto na PEmáx, o que pode indicar que independentemente do protocolo utilizado, a EDET causa efeitos significativos na modificação tanto da força muscular inspiratória quanto expiratória.

Cabe salientar que a força muscular respiratória foi a variável escolhida para observar o efeito da EDET, pois esse recurso tem efeito específico sobre ela, mais particularmente na força inspiratória, pelo fato de a corrente elétrica objetivar a contração do músculo diafragma. Alguns estudos têm evidenciado a importância da avaliação dessa variável pelas pressões respiratórias máximas na avaliação clínica. Segundo Morgan et al. ${ }^{15}$, a PImáx e a PEmáx têm sido utilizadas para identificar o risco de insuficiência respiratória e predizer a sobrevi$\mathrm{da}$ em pacientes com doenças neuromusculares. Outros autores também relataram que especificamente a redução na PImáx é considerada como um fator preditivo de sobrevida em indivíduos com doença pulmonar obstrutiva crônica $(\mathrm{DOPC})^{16} \mathrm{e}$ insuficiência cardíaca ${ }^{17}$.

Nesse contexto, Araújo et al. ${ }^{18}$ relataram que a redução da força muscular respiratória é reabilitável. Sendo assim, no âmbito da fisioterapia respiratória, há o treinamento muscular respiratório com o threshold, que é convencional para se treinar a força ${ }^{19}$ e há também o recurso eletroterapêutico, específico para os músculos inspiratórios, que é a EDET, a qual pode auxiliar na função e também na melhora da força. Embora a EDET seja um recurso pouco estudado e com poucos dados na literatura científica, tem sido utilizada na prática clínica da fisioterapia, porém sem um protocolo com evidências científicas. Tais aspectos justificaram a realização deste estudo, devido à contribuição para fundamentação de protocolos na prática clínica. Apesar disso, empregamos um protocolo que também já foi explorado por Geddes et al. ${ }^{2}$, comparando

Tabela 2. Pressões respiratórias máximas nas fases pré e pós-intervenção dos grupos Controle, Phrenics e Dualpex

\begin{tabular}{lcccccrcr} 
& \multicolumn{3}{c}{ PImáx } & \multicolumn{3}{c}{ PEmáx } \\
\cline { 2 - 7 } & Pré & Pós & $\%$ & Pré & Pós & $94,3 \pm 19,9$ & 1,8 \\
Controle & $74,3 \pm 12,7$ & $76,4 \pm 14,1$ & 2,7 & $92,9 \pm 20,6$ & $80,7 \pm 19,2$ & 44,7 \\
Phrenics & $52,9 \pm 9,1$ & $69,3 \pm 11,0$ & 32,9 & $57,9 \pm 18,5$ & $117,1 \pm 22,7$ & 60,9
\end{tabular}

Média e desvio padrão; n=7/grupo. EDET: estimulação diafragmática elétrica transcutânea; PImáx: pressão inspiratória máxima; PEmáx: pressão expiratória máxima 
a um outro padronizado por equipamento específico da EDET, o qual também tem sido utilizado na prática fisioterapêutica, mas sem evidências científicas.

Nossos resultados estão de acordo com os dados de estudos recentes ${ }^{5}$, realizados com o Phrenics, para o tratamento fisioterapêutico. Nesse estudo, Peres e Kojina ${ }^{5}$ utilizaram a EDET, associada ao tratamento fisioterapêutico na fase pós-operatória de indivíduos submetidos à revascularização do miocárdio e conseguiram demonstrar um efeito benéfico sobre a força muscular inspiratória. Além disso, esses autores detectaram também alterações em alguns parâmetros espirométricos. De forma semelhante, Forti et al. ${ }^{20}$, ao utilizarem a EDET com o Phrenics associada a fisioterapia respiratória convencional, constataram preservação da força muscular expiratória (PEmáx) no período pós-operatório de pacientes submetidos à cirurgia bariátrica, situação em que normalmente essa força é diminuída.

É importante destacar que, apesar de a EDET ter sido destinada especificamente para o músculo diafragma, os músculos expiratórios também apresentaram melhora de sua força. Isso ocorreu, possivelmente, devido à sobreposição da região estimulada. Tal aspecto já havia sido relatado por Durigan et al. ${ }^{21}$ que, ao aplicarem a estimulação elétrica no músculo sóleo de animais, obtiveram efeito no músculo tibial anterior. Uma das justificativas foi o campo elétrico gerado, concluindo que a alta densidade da corrente aplicada deve ter gerado um amplo campo elétrico, suficiente para estimular ambos os compartimentos, posterior (músculo sóleo estimulado diretamente) e anterior (músculo tibial anterior). Considerando que para a estimulação diafragmática, necessariamente a corrente elétrica estimula também a parede abdominal, pela localização dos eletrodos, possivelmente isso justifique o aumento também da força muscular expiratória.

$\mathrm{Na}$ utilização de protocolos de estimulação elétrica, um fator importante é a frequência. Por essa razão, optamos por $30 \mathrm{~Hz}$ em ambos os protocolos, que é adequada para estimular as fibras rápidas e, assim, melhorar a força muscular. Tanto o protocolo de Geddes et al. ${ }^{2}$, quanto nos estudos que utilizam o Phrenics, preconiza-se $30 \mathrm{~Hz}$. Estudos mais antigos como o de Savage ${ }^{22}$ mostraram que as faixas de frequência utilizadas na estimulação de nervos motores estão entre 10 e $50 \mathrm{~Hz}$. Os poucos estudos que são encontrados na literatura envolvendo a EDET em músculos de seres humanos utilizaram $30 \mathrm{~Hz}$ para melhorar a força muscular respiratória ${ }^{5,18}$, motivo pelo qual essa frequência foi selecionada.
Em estudo com animais, Cancelliero et al. ${ }^{23}$ e Costa et al. ${ }^{24}$ detectaram que a EDET, utilizada numa frequência que estimula as fibras rápidas $(50 \mathrm{~Hz})$ melhorou tanto condições energéticas da musculatura respiratória ${ }^{23}$, quanto promoveu alteração no tipo de fibra muscular, com redução da fibra tipo I e aumento da fibra IID $^{24}$. Todos esses dados vêm reforçando a necessidade da busca de parâmetros da corrente elétrica, pois, com isso, a prática clínica estará subsidiada com técnicas seguras e eficientes, além de cientificamente comprovadas.

Há que se considerar a limitação deste estudo, pois o mesmo foi realizado com uma amostra composta somente de mulheres, e sem qualquer comprometimento da saúde respiratória. Além disso, não foram controladas as práticas de exercícios físicos o que, de certa maneira, pode ter interferido no desempenho ou resultado final, embora o estudo tenha sido randomizado. Com isso, fica a sugestão e justificativa para o desenvolvimento de novos estudos, com controle de tais aspectos e exploração mais detalhada da técnica de EDET, que se mostrou eficaz no aumento da força muscular respiratória.

\section{CONCLUSÃO}

Pode-se concluir que não há diferença nos dois protocolos da EDET, pois os dois promoveram aumento das pressões respiratórias máximas, que refletem a força muscular respiratória, confirmando nossa hipótese de que esse recurso pode ser utilizado, com segurança, na prática da fisioterapia respiratória. Porém, como clinicamente o protocolo de Geddes et al. ${ }^{2}$ gerou maior variação de força que o Phrenics, estudos com tamanho amostral maior poderiam ser realizados.

\section{AGRADECIMENTOS}

Quark ${ }^{\circledR}$ Produtos Médicos, voluntários e ao suporte financeiro (PNPD - CNPq: Processo: 151755/2008-6).

\section{REFERÊNCIAS}

1. Hon EH, Hulme GW. An electronic resuscitator for possible use in asphyxia neonatorum. Yale J Biol Med. 1958;31(2):57-73. 
2. Geddes LA, Voorhees WD, Bouland JD. Optimum stimulus frequency for contracting the inspiratory muscle with chest-surface electrodes to produce artificial respiration. Ann Biomed Eng. 1990;18(1):103-8.

3. Cuello AF, Masciantonio L, Mendoza SM. Estimulation diafragmatica eletrica transcutanea. Med Intensiva. 1991:8(4):194-202.

4. Costa D, Forti EMP, Barbalho-Moulim MC, Rasera Jr. I. Study on pulmonary volumes and thoracoabdominal mobility in morbidly obese women undergoing bariatric surgery, treated with two different physical therapy methods. Rev Bras Fisioter. 2009; 13(4):294-300.

5. Peres P, Kojina TY. Uso de eletroestimulação transcutânea diafragmática em pós-operatório de revascularização do miocárdio. Saúde Pesq. 2009:2(1):53-7.

6. Geddes LA, Voorhees WD, Babbs CF. Electroventilation, Proc. 5th Purdue Conf. CPR \& Defibrillation. Am J Emerg Med. 1985;3(4):337-9.

7. Geddes LA, Voorhees WD, Lagler R, Riscili C, Foster K, Bourland JD. Electrically produced artificial ventilation. Med Instrument. 1988;22(5):263-71.

8. Geddes LA, Simmons A. Artificial respiration in the dog by percutaneous bilateral phrenic nerve stimulation. Am J Emerg Med. 1991;9(6):527-9

9. Ross EZ, Nowicky AV, McConnell AK. Influence of acute inspiratory loading upon diaphragm motor-evoked potentials in healthy humans. J Appl Physiol. 2007;102(5):1883-90.

10. Sobush DC, Dunning M 3rd. Assessing maximal static ventilatory muscle pressures using the "bugle" dynamometer. Suggestion from the field. Phys Ther. 1984;64(11):1689-90.

11. Camelo JS, Terra Filho J, Manço JC. Pressões respiratórias máximas em adultos normais. J Pneumol. 1985;11(4):181-4.

12. Souza RB. Pressões respiratórias estáticas máximas. J Pneumol. 2002:28(3):155-65.

13. Neder JA, Andreoni S, Lerario MC, Nery LE. Reference values for lung function tests: II. Maximal respiratory pressures and voluntary ventilation. Braz J Med Biol Res. 1999;32(6):719-27.
14. Forti EMP, Pachani GP, Montebelo MIL, Costa D. Eletroestimulação diafragmática transcutânea em indivíduos saudáveis. Fisioter Bras. 2005;6(4):261-4.

15. Morgan RK, McNally S, Alexander M, Conroy R, Hardiman O, Costello RW. Use of Sniff nasal-inspiratory force to predict survival in amyotrophic lateral sclerosis. Am J Respir Crit Care Med. 2005;171(3):269-74.

16. Gray-Donald K, Gibbons L, Shapiro SH, Macklem PT, Martin JG. Nutritional status and mortality in chronic obstructive pulmonary disease. Am J Respir Crit Care Med. 1996;153(3):961-6.

17. Frankenstein L, Meyer FJ, Sigg C, Nelles M, Schellberg D, Remppis A, et al. Is serial determination of inspiratory muscle strength a useful prognostic marker in chronic heart failure? Eur J Cardiovasc Prev Rehabil. 2008;15(2):156-61.

18. Araújo TL, Resqueti VR, Bruno S, Azevedo IG, Dourado Jr ME, Fregonezi G. Respiratory muscle strength and quality of life in myotonic dystrophy patients. Rev Port Pneumol. 2010;16(6):892-8.

19. Hostettler S, Illi SK, Mohler E, Aliverti A, Spengler CM. Chest wall volume changes during inspiratory loaded breathing. Respir Physiol Neurobiol. 2011;175(1):130-9.

20. Forti E, Ike D, Barbalho-Moulim M, Rasera Jr I, Costa D. Effects of chest physiotherapy on the respiratory function of postoperative gastroplasty patients. Clinics. 2009;64(7):683-9.

21. Durigan JLQ, Cancelliero KM, Bosi PL, Delfino GB, Montebelo MIL, Guirro RRJ, et al. Metabolic and morphometric alterations inherent to neuromuscular electric stimulation in the antagonist muscle submitted to ankle joint immobilization. Braz Arch Biol Technol. 2009;52(1):85-91.

22. Savage B. Interferencial therapy, faber and faberlimitd. London: Faber and Faber; 1984.

23. Cancelliero KM, Costa D, Silva CA. Transcutaneous electrical stimulation of the diaphragm improves the metabolic conditions of respiratory muscles in rats. Rev Bras Fisioter. 2006:10(1):59-65.

24. Costa D, Cancelliero KM, Campos GE, Salvini TF, Silva CA. Changes in types of muscle fibers induced by transcutaneous electrical stimulation of the diaphragm of rats. Braz J Med Biol Res. 2008;41(9):809-11. 